\title{
Helicobacter pylori: preying on SIVA for survival in the stomach
}

\author{
José B. Sáenz' and Jason C. Mills ${ }^{1,2,3}$ \\ 'Division of Gastroenterology, Department of Internal Medicine, ${ }^{2}$ Department of Developmental Biology, and ${ }^{3}$ Department of Pathology and Immunology, Washington University in St. Louis School of \\ Medicine, St. Louis, Missouri, USA.
}

\begin{abstract}
Infection with the Gram-negative bacterium Helicobacter pylori remains the most important modifiable risk factor for the development of gastric cancer a leading cause of cancer-related deaths worldwide. How the interactions between $H$. pylori and its host shape the gastric environment during chronic infection warrants further investigation. In this issue of the $J C I$, Palrasu et al. used human cell lines and mouse models to provide mechanistic insight into $H$. pylori's ability to delay apoptosis in gastric epithelial cells by actively driving the degradation of a proapoptotic factor, SIVA1. Their findings suggest that promoting the survival of gastric epithelial cells has implications not only for $\boldsymbol{H}$. pylori pathogenesis but for host tumorigenesis.
\end{abstract}

\section{Helicobacter pylori overcomes a myriad of host defense mechanisms}

$H$. pylori is one of the most prolific human pathogens, affecting approximately half of the global population (1). The success of $H$. pylori as a chronic pathogen relies in large part on its ability to colonize and persist within the hostile gastric environment by overcoming a myriad of host defense mechanisms, including peristalsis and, most notably, stomach acid. Like other chronic pathogens, H. pylori has fine-tuned its interactions with its host, sufficiently modifying its environment to survive, yet tempering activation of the host inflammatory response to persist. As a result of alterations in host homeostasis, $H$. pylori increases oncogenic risk, with $H$. pylori infection being the single most important factor for the development of gastric cancer (2). H. pylori-derived virulence factors increase cancer risk by inducing chronic, recurrent inflammation while undermining intracellular host defense mechanisms and actively driving changes in epithelial cell proliferation, differentiation, and survival $(3,4)$.

In this issue of the JCI, Palrasu et al. (5) provide detailed, mechanistic evidence to illustrate one method by which H. pylori hijacks arguably the most basic, innate epithelial defense, apoptosis. The authors demonstrated that $H$. pylori infection of human gastric epithelial cells led to downregulation of the proapoptotic factor, SIVA1 (5). SIVA1 promotes CD27and $\mathrm{T}$ cell receptor-mediated apoptosis and inhibits $\mathrm{Bcl}-\mathrm{x}(\mathrm{L})$ antiapoptotic activity during cellular stress (6-8). Specifically, $H$. pylori infection promoted the proteasomal degradation of SIVA1 by activating a ubiquitin ligase and X-linked inhibitor of apoptosis, XIAP. Importantly, H. pylori's antiapoptotic effects through SIVA1 are mediated by CagA, an $H$. pylori-specific virulence factor that is delivered into host cells by a type IV secretion system and that influences multiple host intracellular processes (9). CagA directly regulates SIVA1 stability in vitro by stimulating its degradation. Accordingly, individuals infected with cagA-positive strains of $H$. pylori showed increased XIAP phosphorylation (the activated form of the protein) and decreased SIVA1 compared with uninfected individuals or those infected with cagA-negative strains (5). This article thus identifies yet another key function of the versatile CagA protein (10), in this case promoting $H$. pylori pathogenesis by subverting established host apoptotic defense mechanisms (5).

\section{Apoptosis in the stomach}

The Palrasu et al. study (5) forces us to reconsider the evolutionary tit-for-tat that exists between $H$. pylori and the human stomach. $H$. pylori colonizes the stomach predominantly as an extracellular pathogen (11), so what would be the benefit to the bacterium in delaying apoptosis and promoting the survival of epithelial cells in which it does not primarily reside? One possibility is that these host epithelial cells contribute to a mucinous, extracellular matrix that provides attachment sites for $H$. pylori as well as shelters the bacteria from the highly acidic gastric lumen (12). Apoptosis remains an effective means for the host to discard these harboring cells and limit $H$. pylori's epithelial foothold. $H$. pylori, not to be outdone, might have developed its own arsenal to specifically overcome apoptosis and enhance its colonization. In addition to blocking apoptosis, a previous study showed that $H$. pylori also relies on CagA to promote and maintain a proliferative state that enhances colonization (13).

From the host perspective, apoptosis in the stomach serves a critical function beyond simply restraining bacterial colonization. Apoptosis clears injured epithelial cells and limits ongoing cellular damage, which may be critical because $H$. pylori infection produces a characteristic genotoxicity, with DNA damage at specific regions in the host genome, namely in transcribed regions and proximal to telo- 
meres (14). H. pylori has also been shown to inhibit host DNA repair mechanisms (15). In addition to DNA damage, persistent injury to gastric epithelial cells results in repeated rounds of cellular reorganization and proliferation, as part of an innate and conserved intracellular response to repair the gland (16). With each round of proliferation, these metaplastic cells can accumulate mutations that can predispose to the development of cancer. $H$. pylori could directly contribute to this cyclical hit model of tumorigenesis (17) by promoting survival of metaplastic cells harboring DNA damage or, specifically, mutations in key growth or differentiation-regulating genes. The authors show that the effect of H. pylori on SIVA1 actively limits the host response to DNA damage, independently of a critical regulator of the DNA damage response, p53 (18). Taken together, Palrasu et al. (5) lend further support for $H$. pylori's carcinogenic potential (19) through the CagA-mediated downregulation of apoptotic mechanisms that would otherwise weed out mutant cells.

\section{Chronic H. pylori infection downregulates SIVA1 expression}

While Palrasu and colleagues primarily focused on $H$. pylori's effects on SIVA1 in a human gastric cancer cell line (5), we note that the cell-specific expression of SIVA1 in vivo could have substantial implications for $H$. pylori pathogenesis and host tumorigenesis. For example, Siva1 was largely absent from the mucus-secreting foveolar/pit cells in mice. Broadly speaking, delaying apoptosis in this population of epithelial cells may confer an advantage to $H$. pylori in the more acute stage of infection, when $H$. pylori must gain a foothold within the infected gland (13), but it would carry little to no carcinogenic risk to the host, as these rapidly cycling cells are continually extruded from the tops of gastric glands into the gastric lumen. However, Siva1 was highly and almost exclusively expressed in chief cells at the bases of gastric glands within the gastric corpus, the acid-secreting region of the stomach, and was subsequently lost following chronic $H$. pylori infection (5). Injured gastric corpus glands can undergo a reorganization, characterized by the reprogramming of chief cells at the gland base into a population of proliferative, metaplastic cells (20). Downregulation of chief cell-specific SIVA1, therefore, could promote the survival of chief cells, representing one method by which $H$. pylori might increase oncogenic risk, since long-lived chief cells are prone to cyclical reprogramming events that can lead to chronic accumulation of DNA damage and an increased potential for neoplastic transformation $(17,20)$. Moreover, the reprogramming of chief cells promotes $H$. pylori's chronic colonization and expansion throughout the stomach (21).

While this study by Palrasu et al. implicates SIVA1 as a critical component of $H$. pylori pathogenesis and as a key checkpoint in the progression to gastric cancer following chronic $H$. pylori infection (5), questions remain. Does the degradation of SIVA1 mark the evolution toward a preneoplastic state during chronic $H$. pylori infection? Can decreased SIVA1 levels portend a poorer prognosis in patients chronically infected with $H$. pylori? What is the mutational burden in cells that have degraded SIVA1? What is the ultimate fate of these cells, and are other host mechanisms in place to trigger apoptosis in the face of decreased SIVA1 levels? This study prompts a deeper understanding into the complex but critical host and microbial mechanisms that regulate the mutational landscape and underlie the gastric preneoplastic milieu.

\section{Acknowledgments}

JCM is supported by National Institute of Diabetes and Digestive and Kidney Diseases (NIDDK) awards R01DK105129, R01DK094989, and 5P30DK052574. JBS is supported by NIDDK award K08DK122116-0 and an American Gastroenterological Association Gastric Cancer Foundation Research Scholar Award.

Address correspondence to: José B. Sáenz and Jason C. Mills, Washington University in St. Louis School of Medicine, 660 South Euclid Avenue, St. Louis, Missouri 63110, USA. Phone: 314.362.4213; Email: saenzj@wustl.edu (JBS); jmills@wustl. edu (JCM).

1. Hooi JKY, et al. Global prevalence of Helicobacter pylori infection: systematic review and meta-analysis. Gastroenterology. 2017;153(2):420-429.

2. Plummer M, de Martel C, Vignat J, Ferlay J, Bray
F, Franceschi S. Global burden of cancers attributable to infections in 2012: a synthetic analysis. Lancet Glob Health. 2016;4(9):e609-e616.

3. Amieva M, Peek RM. Pathobiology of Helicobacter pylori-induced gastric cancer. Gastroenterology. 2016;150(1):64-78.

4. Franco AT, et al. Regulation of gastric carcinogenesis by Helicobacter pylori virulence factors. Cancer Res. 2008;68(2):379-387.

5. Palrasu M, et al. Bacterial CagA protein compromises tumor suppressor mechanisms in gastric epithelial cells. J Clin Invest. 2020;130(5):2422-2434.

6. Prasad KV, et al. CD27, a member of the tumor necrosis factor receptor family, induces apoptosis and binds to Siva, a proapoptotic protein. Proc Natl Acad Sci U S A. 1997;94(12):6346-6351.

7. Xue L, et al. Siva-1 binds to and inhibits BCL-X(L)-mediated protection against UV radiation-induced apoptosis. Proc Natl Acad Sci U S A. 2002;99(10):6925-6930.

8. Chu F, et al. The Siva-1 putative amphipathic helical region (SAH) is sufficient to bind to BCL$\mathrm{XL}$ and sensitize cells to UV radiation induced apoptosis. Apoptosis. 2004;9(1):83-95.

9. Odenbreit S, Püls J, Sedlmaier B, Gerland E, Fischer W, Haas R. Translocation of Helicobacter pylori CagA into gastric epithelial cells by type IV secretion. Science. 2000;287(5457):1497-1500.

10. Hatakeyama M. Helicobacter pylori CagA and gastric cancer: a paradigm for hitand-run carcinogenesis. Cell Host Microbe. 2014;15(3):306-316.

11. Dubois A, Borén T. Helicobacter pylori is invasive and it may be a facultative intracellular organism. Cell Microbiol. 2007;9(5):1108-1116.

12. Tan S, Tompkins LS, Amieva MR. Helicobacter pylori usurps cell polarity to turn the cell surface into a replicative niche. PLoS Pathog. 2009;5(5):e1000407.

13. Mimuro H, et al. Helicobacter pylori dampens gut epithelial self-renewal by inhibiting apoptosis, a bacterial strategy to enhance colonization of the stomach. Cell Host Microbe. 2007;2(4):250-263.

14. Koeppel M, Garcia-Alcalde F, Glowinski F, Schlaermann P, Meyer TF. Helicobacter pylori infection causes characteristic DNA damage patterns in human cells. Cell Rep. 2015;11(11):1703-1713.

15. Kim JJ, Tao H, Carloni E, Leung WK, Graham DY, Sepulveda AR. Helicobacter pylori impairs DNA mismatch repair in gastric epithelial cells. Gastroenterology. 2002;123(2):542-553.

16. Willet SG, et al. Regenerative proliferation of differentiated cells by mTORC1-dependent paligenosis. EMBO J. 2018;37(7):e98311.

17. Sáenz JB, Mills JC. Acid and the basis for cellular plasticity and reprogramming in gastric repair and cancer. Nat Rev Gastroenterol Hepatol. 2018;15(5):257-273.

18. Sengupta S, Harris CC. p53: traffic cop at the crossroads of DNA repair and recombination. Nat Rev Mol Cell Biol. 2005;6(1):44-55.

19. [no author listed]. Schistosomes, liver flukes and Helicobacter pylori. IARC Working Group on the Evaluation of Carcinogenic Risks to Humans. 
Lyon, 7-14 June 1994. IARC Monogr Eval Carcinog Risks Hum. 1994;61:1-241.

20. Burclaff J, Willet SG, Sáenz JB, Mills JC. Proliferation and differentiation of gastric mucous neck and chief cells during homeostasis and injury-induced metaplasia. Gastroenterology. 2020;158(3):598-609.e5.

21. Sáenz JB, Vargas N, Mills JC. Tropism for spasmolytic polypeptide-expressing metaplasia allows Helicobacter pylori to expand its intragastric niche. Gastroenterology. 2019;156(1):160-174.e7. 